\title{
Acknowledgement to Reviewers of Diversity in 2019
}

Diversity Editorial Office

MDPI, St. Alban-Anlage 66, 4052 Basel, Switzerland

Published: 17 January 2020

The editorial team greatly appreciates the reviewers who have dedicated their considerable time and expertise to the journal's rigorous editorial process over the past 12 months, regardless of whether the papers are finally published or not. In 2019, a total of 258 papers were published in the journal, with a median time to first decision of 16.5 days and a median time from submission to publication of 41 days. The editors would like to express their sincere gratitude to the following reviewers for their generous contribution in 2019:

Aarnio, Katri

Abdul Wahab, Muhammad

Abramoff, Rose

Accoroni, Stefano

Adam, Paul

Aebi, Alex

Agathokleous, Evgenios

Agnese, Jean-Francois

Aguilar, Juan Carlos Villarreal

Ahlrichs, Wilko

Ahmed, Mohammed

Aizpurua, Ostaizka

Alexander, James E.

Alía, Ricardo

Allegrezza, Marina

Allen, Edith

Allgeier, Jake

Amey, Katherine Springer (Kay)

An, Hong

An, Ran

Anastácio, Pedro

Anderson, Michael A.

Anderson, Robert

Andrew M., Zealand

Andrew, Tedder

Angradi, Theodore

Anna, Tomczyk

Antoniadou, Chryssanthi

Aragón, Gregorio

Ariyawansa, Hiran Anjana

Arizza, Vincenzo

Armenteros, Maickel

Arpaia, Mary Lu

Arslan, Muhammad

Auclerc, Apolline
Avens, Larisa

Aylward, Frank O.

Aznar, Francisco Javier

B. Connell, Laurie

Babin, Doreen

Badiane, Arnaud

Baena, Susana

Bagur, Maria

Baker, Ronald

Balestrieri, Alessandro

Ballot, Andreas

Ballvora, Agim

Ban, Yusuke

Baranov, Viktor

Barina, Zoltán

Barrell, Jeffrey

Bates, John

Battisti, Corrado

Baumann, Justin

Baumung, Roswitha

Bazzicalupo, Marco

Beck, Andreas

Bell, F. Wayne

Bell, Tom W.

Bellgrove, Alecia

Belmonte, Genuario

Benda, Petr

Benevenuto, Juliana

Benjamin, Pierce

Bento-Gonçalves, António

Bergmann, Luke

Bernardi Aubry, Fabrizio

Bernhardt, Peter

Berny Mier Y Teran, Jorge Carlos

Bertolini, Camilla 
Betti, Federico

Beyer, Marco

Bik, Holly M.

Bird, Chris

Blackburn, David C.

Blamires, Sean

Bledzki, Leszek A.

Blom, Mozes

Bode, Antonio

Bohner, Andreas

Bombi, Pierluigi

Bonari, Gianmaria

Bond, Monica L.

Bonnin, Rémy

Booth, David

Bos, Arthur R.

Bosi, Sara

Bosso, Luciano

Bothwell, John

Bowen, Gabriel J.

Bowsher, Julia H.

Brandyk, Andrzej

Brannelly, Laura

Brill, Richard W.

Brim-DeForest, Whitney

Brodie, Jon

Brodschneider, Robert

Brooks, Ronald

Brosset, Pablo

Broussard, Gregory

Brouwer, Adam

Brown, Charles R.

Brown, Donald

Brown, Jason Lee

Brundu, Giuseppe

Brunialti, Giorgio

Brusca, Richard C.

Brygadyrenko, Viktor V.

Bryja, Josef

Bubici, Giovanni

Bucekova, Marcela

Bugnot, Ana

Burczyk, Jarosław

Burger, Pamela

Burnett, Stephen

Burns, John

Burritt, James B.

Byers, James

C. Brustolin, Marco

Cadena, Edwin

Cairns, Kylie

Calero, Sara
Calò, Antonio

Campbell, Ewan

Campos, Maria Doroteia

Caneva, Giulia

Cannizzo, Zachary J.

Cannon, Jeffery B.

Cano, Jorge

Cao, Yang

Carapelli, Antonio

Cardenas-Garcia, Stivalis

Carney, Joseph

Čarni, Andraž

Carotenuto, Ylenia

Carr, John L

Carter, Jacoby

Carvalho, Filipe

Castillo, Karl

Catenazzi, Alessandro

Cayuela, Luis

Cermeño, Pedro

Česonienè, Laima

Chadwick, Nanette

Chapman, Daniel

Chauhan, Garima

Chen, Chaolun

Chen, Ji

Chłond, Dominik

Chmura, Damian

Christou, Epaminondas D.

Chroni, Antonia

Chylarecki, Przemysław

Ciach, Michał

Cicchetti, Giancarlo

Cisterna, Pedro

Claramunt, Santiago

Claude, Julien

Clavijo-McCormick, Andrea

Clay, Keith

Cleavitt, Natalie L.

Clevenger, Anthony

Colla, Sheila R.

Colman, Dan

Copp, Gordon

Corby-Harris, Vanessa Louise

Cornils, Astrid

Coro, Gianpaolo

Corrado, Giandomenico

Cortés, Andrés J.

Costantini, Federica

Cowan, Zara-Louise

Cranston, Peter

Crego, Ramiro Daniel 
Crook, David

Croshaw, Dean

Crowley, Brooke

Cunha-Lignon, Marilia

Cycoń, Mariusz

Da Silva, Pedro

Dal Forno, Manuela

Daly, Kristian

Danihlík, Jiří

Dari, Biswanath

Darras, Kevin

Davidova, Irene

Davy, Christina

De Almeida, André M.

De Bruijn, Irene

De la Riva, Ignacio

De Maayer, Pieter

De Oliveira, Hernani Fernandes Magalhães

DeBiasse, Melissa

Debnath, Samir

Del Vecchio, Silvia

Delgado Bermejo, Juan Vicente

DellaSala, Dominick

Dembowska, Ewa Anna

Dethier, Megan

Diamond, Antony

Dimante-Deimantoviča, Inta

Dimkić, Ivica

Dobrowolska, Dorota

Dodd, Richard S.

Dorchin, Netta

Dowd, Patrick F.

Drava, Giuliana

Drees, Claudia

Driggers, William B.

Ducatez, Simon

Duchene, David

Dufresne, France

Dunlap, Christopher

Dutto, Moreno

Easy, Russell

Ebrahimi, Aziz

Edwards, Patrick

Efremenkova, Olga V.

Ellis, Christopher

Ellison, Aaron M.

Eppley, Timothy M.

Ericson, Per G.P.

Eriksson, Susanne

Erikstad, Lars

Escalona, Tibisay

Etienne, Lucie
Eurich, Jacob

Faillettaz, Robin

Fan, Qinghai

Faraji, Farid

Feás, Xesús

Fell, Richard

Ferencakovic, Maja

Fickert, Thomas

Fischer-Kowalski, Marina

Fišer, Žiga

Flores-Félix, J.D.

Fois, Mauro

Foley, Melissa M.

Ford, Mark

Forsberg, Kevin

Forster, Rodney

Framenau, Volker

Francesco, Smedile

Franco, Miguel

Frank, Alexander

Franklin, Don

Fransen, Charles

Fray, Martin

Fredericq, Suzanne

Freedberg, Steven

Freeman, Christopher J

Frommel, Andrea Y.

Fthenakis, George

Fukunaga, Atsuko

Gabriel, Rosalina

Gajdzik, Laura

Gajger, Ivana Tlak

Gałązka, Anna

Galvani, Emerson

Ganzhorn, S.M.

Garbayo, Inés

García Del Barrio, José

Garcia-Ayllon, Salvador

García-Martínez, María Del Carmen

Garcia-Porta, Joan

García-R, Juan Carlos

Garofalo, Luisa

Gauthier, Martin-Michel

Gazda, Anna

Gazzola, Andrea

Geiser, Linda

Gentili, Rodolfo

George, Kai Horst

Gerlach, Gabriele

Ginja, Catarina

Giordani, Paolo

Giovannetti, Manuela 
Giraldo, Alan

Girondot, Marc

Gisbert, Enric

Gnanvossou, Desire

Goberville, Eric

Godschalx, Adrienne L.

Goleman, Małgorzata

Gollmann, Günter

Gonzalez, Brett

Goodbody-Gringley, Gretchen

Gordeev, Lya I.

Gornall, Richard

Goulet, Denis

Goyache, Félix

Grabner, Daniel S.

Grabowska, Magdalena

Graf, W.

Granzow-de La Cerda, Íñigo

Grau-Andrés, Roger

Gray, Murray

Greenslade, Penelope

Gregorič, Matjaž

Griffiths, Robert

Gross, Elisabeth Maria

Grossman, Jake Joseph

Guarnaccia, Vladimiro

Guidi Nissim, Werther

Gülay, Arda

Gupta, Vijai Kumar

Guselnikov, Sergey

Gweon, Hyun Soon

Hale, Amanda

Halstead, Brian

Hämäläinen, Aino

Hammock, Bruce G.

Hanslin, Hans Martin

Harnisz, Monika

Haroun, Ricardo

Harris, James

Harrison, Hugo

Harrison, Joshua G.

Harvey, Mark

Hawes, Ian

Hawes, Joseph E.

Hawkins, Stuart

Hay, Mark E.

Hayes, Kenneth A.

Hays, Graeme

Hegarty, Matthew

Heneberg, Petr

Hermida, Margarida

Hernandez-Gomez, Obed
Heyward, Andrew

Hibbitts, Toby

Hilty, Jodi

Hinojosa, M Belen

Hodder, Kathy

Hoeksema, Bert

Hoffmann, Samuel

Holland, Nicholas D.

Holovachov, Oleksandr

Hong, Ray

Hong, Yan

Honka, Johanna

Hooper, Daniel

Hormiga, Gustavo

Hotos, George N.

Houliez, Emilie

Hsu, Te-Hua

Huang, Da-Ji

Huerlimann, Roger

Huey, Joel

Hughes, Daniel F.

Huijser, Marcel P.

Hutson, Kate

Huvet, Arnaud

Hyndes, G.A.

I De Lanuza, G. Pérez

Ihle, Kate E

Illera, Juan Carlos

Irestedt, Martin

Isermann, Maike

Islebe, Gerald Alexander

Iso-Touru, Terhi

Ivankovic, Ante

Jandl, Robert

Jarret, Robert L.

Jeffs, Andrew

Jehle, Robert

Jelaska, Sven

Jensen, Kevin B.

Jersabek, Christian

Jiang, Xiaoben

Jiggins, Chris

Johansson, Eva

Johnston, J. Spencer

Jones, Darryl

Jordán, Ferenc

Jorrin, Beatriz

Joseph, Leo

Jung, Martin

Justine, Jean-Lou

Kafle, Arjun

Kakareko, Tomasz 
Kalwasińska, Agnieszka

Kamaldinov, Evgeniy

Kamijo, Takashi

Kanavillil, Nandakumar

Kane, Evan

Kang, Sanghoon

Karayanni, Hera

Kariyat, Rupesh

Katam, Ramesh

Kawakami, Takeshi

Keller, Aimee

Kerbiriou, Christian

Kerbis-Peterhans, Julian C.

Kerschbaumer, Michaela

Kezic, Nikola

Kim, Haeng-hoon

King, Sarah R.B.

Király, Gergely

Kirkman, Hugh

Knisley, Charles Barry

Kobak, Jarosław

Kobayashi, Donald

Kobiv, Yuriy

Koblmüller, Stephan

Koide, Dai

Kok, Philippe J. R.

Kolesar, Sarah

Kolicka, Małgorzata

Konstantin, Milto

Kooij, Pepijn W.

Kooistra, Wiebe H.C.F.

Kovačević, Goran

Krauel, Jennifer J.

Krauze-Gryz, Dagny

Krist, Amy

Krupa, Elena

Kubátová, Alena

Kubiak, Dariusz

Kuczyńska-Kippen, Natalia

Kuhlwilm, Martin

Kuitems, Margot

Kulichová, Jana

Kull, Tiiu

Kuraku, Shigehiro

Kurosawa, Norio

Labisko, Jim

Labokas, Juozas

Lafferty, Diana

Laghetti, Gaetano

Laloë, Jacques-Olivier

Landis, Jacob

Langlois, Tim
Lanubile, Alessandra

Lapointe, Line

Latombe, Guillaume

Laurino, Daniela

LAVOIE, KATHLEEN

Lawniczak-Malińska, Agnieszka E.

Layton, Kara

Lecours, Vincent

Legal, Luc

Lehtinen, Richard

Lehtonen, Samuli

Leila, Korpela

Lendemer, James

Lenzner, Bernd

Leonard, Jeremy

Leplat, Johann

Leroy, Gregoire

Leroy, Gregoire

Lewinski, Nastassja A.

Lewis, Keith

Lewis, Matthew

Librado, Pablo

Liedvogel, Miriam

Liversage, Kiran

Lobel, Phillip S.

Loiko, Sergey V.

Lomakina, Anna V.

Long, Jim

Longa, Claudia Maria Oliveira

López-Calderón, Cosme

Loppi, Stefano

Lorite, Juan

Louda, William

Loughman, Zach

Lovelace, David

Łubek, Anna

Luckenbach, Mark W.

Lukac, Martin

Lynch, Heather J.

Lynch, Scott M.

Lyons, Russell

Ma, Guojia

Maccherini, Simona

Macpherson, Maggie P.

Maduna, Simo

Maggioni, Davide

Maire, Olivier

Maki, James

Maki-Tanila, Asko

Makkonen, Jenny

Malatesta, Luca

Malatinszky, Ákos 
Mammola, Stefano

Manenti, Raoul

Manninen, Sirkku

Marchini, Agnese

Marcoleta, Andrés E.

Marcos, Rubal

Maria Eugenia Leone, Gold

Mariottini, Paolo

Marko, Michelle D.

Martellos, Stefano

Martínez, Isabel

Martínez, Luis Carlos

Martins, Gustavo M.

Marvaldi, Adriana Elena

Maschinski, Joyce M.

Mateus, Octávio

Mathai, Prince

Matos, Paula

Matteucci, Enrica

Matttila, Tapani

Mays, Herman

Mazei, Yuri A.

McAlpine, Donald F.

McCarthy, Daniel P.

McFarlane, Donald A.

McGill, Rona

McKinnon, A. David

McWhorter, Todd

Melcher, Ulrich

Melcher, Ulrich

Melis, Claudia

Melo, Bruno F.

Melosik, Iwona

Melzner, Frank

Mende, Daniel R

Mendes, Thiago

Messyasz, Beata

Meunier, Cédric L.

Meyer, Jean-Yves

Meziti, Alexandra

Mezösi, Gábor

Michaux, Johan

Michel, Nicole L.

Mickan, Bede

Mikusinski, Grzegorz

Milanovich, Joe

Milenovic, Milan

Millán, Andrés

Minton, Russell

Mirza, Babur S.

Mirza, Babur S.

Mittapelly, Priyanka
Mohamed, Alburaki

Mojib, Nazia

Moldovan, Oana Teodora

Montano, Simone

Montoro Girona, Miguel

Moody, Eric K

Moore, Paul

Morais, Pedro

Morales-Serna, Francisco Neptalí

Moreno-Rueda, Gregorio

Morri, Carla

Mossialos, Dimitris

Motzo, Rosella

Muggia, Lucia

Muir, Anna P.

Muir, Paul

Muldoon, Kathleen M.

Muñoz-Gómez, Sergio A.

Mutshinda, Crispin

Nadyeina, Olga

Nafus, Melia

Nagamine, Yoshitaka

Nagle, Roy

Nascimbene, Juri

Nedoluzhko, Artem Valerievich

Neilson, Roy

Nelson, David

Neuenkamp, Lena

Nikouli, Eleni

Nilforooshan, Mohammad Ali

Noguchi, Tamao

Normark, Benjamin

Nowicki, Robert

Nozawa, Yoko

O. Rogers, Scott

Oberbauer, Anita M.

Obertegger, Ulrike

Oldenbroek, JK (Kor)

Oliveira, Cláudia

Olli, Kalle

Ondreičková, Katarína

Onuma, Manabu

Oono, Ryoko

Östman, Örjan

Ozturk, Cahit

Pabis, Krzysztof

Pagano, Marcela

Pagnotta, Mario A.

Pagou, Kalliopi

Pallavicini, Alberto

Papale, Maria

Parajuli, Saroj 
Parrino, Vincenzo

Parsons, Darren Michael

Patricio, Ana Rita

Pattison, Zarah

Paver, Sara F.

Pavloudi, Christina

Pearson, Ryan M.

Pereira, Leonel

Pereira, Paulo

Pereira, Tiago

Pereira-Lorenzo, Santiago

Pereira-Silva, Erico Fernando

Perkins, Lora

Péron, Guillaume

Petitpas, Christian M.

Piazzi, Luigi

Pica, Daniela

Piel, Alexander

Pierson, James

Pieszka, Marek

Pinheiro, Hudson T.

Pinou, Theodora

Pinto, Sandra

Pisanu, Benoît

Pita, Lucia

Pocklington, Jacqui

Porporato, Marco

Porter, Raymond

Portillo López, Amelia

Pothoven, Steven

Poulicek, Mathieu E.A.

Pratchett, Morgan S.

Přívětivý, Tomáš

Prowant, Lisa

Pucciarelli, Sandra

Pulis, Eric

Purcell, Kathryn L.

Pusceddu, Antonio

Pyron, R. Alexander

Pyzik, Adam

Qiu, Jian-Wen

Queenborough, Simon

Queiros, João

Quero, Grazia

Raatz, Michael

Ragkos, Athanasios

Raikhy, Gaurav

Rainho, Ana

Randall, Carly J.

Randhawa, Imtiaz

RASCONI, SERENA

Rassner, Sara
Ratkowsky, David

Raudabaugh, Daniel

Redondo-Nieto, Miguel

Reed, David W.

Reich, Marlis

Reichard, Martin

Reid, Brendan

Reid, Rachel E. B.

Reilly, Sean B.

Reimer, James

Requier, Fabrice

Rescia, Alejandro

Ribbons, Relena

Richard, Benjamin

Rico, Jose M

Roberts, Dale

Roberts, Thomas Edward

Robinson, Leonie

Robuchon, Marine

Roccotelli, Angela

Roces-Diaz, Jose V.

Rocha, Luiz

Roche, Ronan

Rodríguez-Peña, Rosa A.

Roe, John

Roff, George

Roger, Eduardo M. García

Roles, Angela

Roman, Michael R.

Romero-Calcerrada, Raúl

Ronconi, Robert

Roosenburg, Willem

Rosati, Leonardo

Rose, Jonathan

Rosenbaum, Peter A.

Rossaro, Bruno

Rovero, Francesco

Rowe, Rebecca

Rowland, Freya

Rowley, Jodi

Roy, Christian

Roy, Helen

Rulifson, Roger

Ryabov, Eugene

Ryan, Emma J.

Ryan, Peter

Saha, Surya

Saint-Paul, Ulrich

Saitoh, Kenji

Sakamoto, Makaki

Sakar, Germán

Samimi-Namin, Kaveh 
Sanchez-Bayo, F

Sandulli, Roberto

Sanfilippo, Marilena

Santelmann, Mary

Sarabeev, Volodimir

Sarti, Francesca Maria

Sato, Hiroshi

Scalici, Massimiliano

Scasta, John Derek

Scheffers, Sander

Scherrer, Daniel

Schlesinger, Matthew D.

Schoenrock, Kathryn M.

Schofield, Gail

Schon, Nicole

Schulze, Anja

Scofield, R. Paul

Scott, Angela Bullanday

Scotton, Michele

Sedlock, Jodi L.

Seitz, Rochelle

Selas, Vidar

Selden, Paul

Sequeira, Ana Martins

Serra, Sónia Raquel Quinás

Seymour, Mathew

Sharma, Jyotsna

Sharma, Lav

Shazib, Shahed Uddin Ahmed

Shiels, Aaron B.

Shivarudrappa, Shivakumar K.

Shoemaker, Kevin

Simon Delso, Noa

Skorupski, Jakub

Škvorc, Željko

Sladonja, Barbara

Slawson, Robin

Sługocki, Łukasz

Smaers, Jeroen

Smeti, Evangelia

Smith, Adam

Smith, Melissa C.

Smith, Robert J.

Smith, Steve

Soanes, Kylie

Sofia Pinto, Ramalho

Solberg, Svein

Sommers, Pacifica

Soriano, Jose Miguel

Sotka, Erik

Soto-Centeno, J. Angel

South, Paul M
South, Paul M.

Søvik, Eirik

Spanbauer, Trisha

Spivak, Marla

Špoljar, Maria

Sponenberg, Phillip

Stachowitsch, Michael

Staentzel, Cybill

Stanek-Tarkowska, Jadwiga

Stanish, Lee

Steen, David A.

Stefano, Schiaparelli

Steinhagen, Dieter

Steinheim, Geir

Stepanek, Joshua G

Stieb, Sara

Streich, Matthew

Striedter, Georg

Strong, Ellen E

Strutzenberger, Patrick

Stuart-Smith, Jemina

Sujetovienè, Gintare

Sullivan, Mark

Summerell, Brett

Sungthong, Rungroch

Szafranek-Nakonieczna, Anna

Szentgyörgyi, Hajnalka

Szymura, Magdalena

Tadesse, Mesfin

Takakura, Kohichi

Takeda, Satoshi

Takumi, Shigeo

Tamplin, Jeff

Tapio, Miika

Taskinen, Sara

Taylor, Brett M.

Terry, Rebecca

Theodoropoulos, Christos

Theriot, Edward

Thibault, Delphine

Thompson, Cassandra

Thomsen, Mads Solgaard

Thomson, Linda

Thurman, Carl Leo

Timmermans, Martijn

Tinya, Flóra

Title, Pascal

Todd, Brian D.

Tökölyi, Jácint

Tollis, Marc

Török, Peter

Torres-Perez, Juan 
Tóthmérész, Béla

Tremblay, Nelly

Tricarico, Elena

Trognitz, Friederike

Tryjanowski, Piotr

Tschinkel, Walter R.

Turco, Ronald F.

Türke, Manfred

Twardowski, Jacek

Tyers, Alexandra

Tzika, Athanasia C.

Ueno, Takatoshi

Umanzor, Schery

Underwood, Robyn

Uwai, Shinya

Vacher, Jean-Pierre

Vallini, Giovanni

Van De Poll, Willem H.

Van Dijk, Peter Paul

Van Dobben, Han F

Van Ee, Benjamin W.

Van Etten, James

Van Marle-Koster, Este

Varela, Zulema

Vasileiadou, Katerina

Vega-Frutis, Rocío

Verbylaitè, Rita

Vercelloni, Julie

Verma, Satish K

Vermeire, Marie-Liesse

Vilizzi, Lorenzo

Vinagre, Catarina

Violeta, Razmaitè

Volk, Gayle

Von May, Rudolf

Wahl, Martin

Wallace, John

Ward, Selina

Warwick, R.M.

Watanabe, Junya

Watson, Jabin

Węgrzyn, Michał

Wemheuer, Bernd

Werner, Johannes
Westerman, Erica

Whitaker, Justine

White, Kristine

White, Kristine N.

Whiteman, John P.

Wieczorek, Karina

William G., Meikle

Williams, Roger

Williams, Stephen

Windig, J.J. (Jack)

Wiseman, Steve

Witt, Christopher C.

Wojtal, Agata Z.

Wonchoe, Lee

Woodhouse, Jason

Worthy, Trevor

Wurdig Roesch, Luiz Fernando

Wurzinger, Maria

Wynne, Michael J.

Wyszkowska, Jadwiga

Xavier, Raquel

Xiao, Chuan (River)

Yamaguchi, Atsushi

Yamano, Hiroya

Yates, Adam Gordon

Yau, Sheree

Yen, Jian

Yip, Bon Ham

Yoshida, Kohta

Yuan, Yuxuan

Zaini, Paulo

Zanolla, Marianela

Zarabska-Bożejewicz, Daria

Zettler, Lawrence W.

Zhang, Chunhua

Zhang, Y. Stacy

Zhu, Baoli

Žiarovská, Jana

Zingel, Priit

Zinovieva, Natalia A.

Zolnerowich, Gregory

Źróbek-Sokolnik, Anna

Zucconi, Laura

(C) 2020 by the authors. Submitted for possible open access publication under the terms and conditions of the Creative Commons Attribution (CC BY) license (http://creativecommons.org/licenses/by/4.0/). 